\title{
Health effects of omega-3,6,9 fatty acids: Perilla frutescens is a good example of plant oils
}

\author{
Mohammad Asif
}

Received: 9 September 2010 /Accepted: 17 January 2011 / Published online: 4 March 2011

(C) The Author(s) 2011. This article is published with open access at Springerlink.com

\begin{abstract}
Perilla frutescens seeds are a good source of polyunsaturated fatty acids (PUFAs). The seeds of perilla are small end globular weight about $4 \mathrm{~g} / 1000$, contained approximately $35-45 \%$ oil. However the leaves are a very poor source of oil, since they contain only $0.2 \%$. In addition, only the seed oil contains the omega 3 fatty acid alpha-linolenic acid (ALA). In comparing to other plant oils, perilla seed oil consistently contains the one of the highest proportion of omega-3 (ALA) fatty acids, at $54-64 \%$. The omega-6 (linoleic acid) component is usually around $14 \%$ and omega-9 (Oleic acid) is also present in perilla oil. These polyunsaturated fatty acids are most beneficial to human health and in prevention of different diseases like cardiovascular disorders, cancer, inflammatory, rheumatoid arthritis etc.
\end{abstract}

Keywords Perilla frutescencs . Polyunsaturated fatty acids · Alpha-linolenic acid · Cardiovascular .

Inflammatory $\cdot$ Rheumatoid arthritis

\section{Introduction}

General description of Perilla frutescencs

Perilla frutescens, is a member of the Lamiaceae/Labiatae family and commonly called perilla. The crop is annual and is native to India and China. Major producing countries of perilla are China, India, Japan, Korea, Thailand, other East

\section{Asif $(\bowtie)$}

Department of Pharmacy,

GRD (PG) Institute of Management \& Technology,

Rajpur Road,

Dehradun 248009, India

e-mail: aasif321@gmail.com
Asian countries. The herb is about $1 \mathrm{~m}$ high with small flowers, a gray-brown fruit, and glossy, downy-haired leaves. Cultivation of the crop is grown from seed and sown in May. Harvesting is usually between the end of September and beginning of October. The applicable parts of perilla plants are the leaves and seeds. There are two main types: red and green perilla. Perilla seeds, also called EBARA seed. This oil is a kind of light yellow clear and transparent liquid, with aromatic odor and slight soluble in ethanol. Major fatty acids of the oil are unsaturated fatty acids like Oleic acid 14-23\%, linoleic acid 11-16\%, linolenic acid 54-64\% (Graph 1). This oil also contains saturated fatty acids $6.7-7.6 \%$. Perilla seeds contain different polyphenols or flavones (rosemarinic acid, luteolin, chrysoeriol, quercetin, catcehin, apegenin and shishonin). Perilla seed oil is used as cooking oil, fuel. It is dry oil used as in paint, varnish and ink manufacturing or as a substitute for linseed oil. The seed cakes are used as animals and birds feed (Gediminas et al. 2008; Talbott and Hughes 2006; Longvah et al. 2000; Borchers et al. 1997; Narisawa et al. 1994).

Source and uses of omega fatty acids

Perilla is used for oil production as a rich source of omega-3 polyunsaturated fatty acids (PUFAs), specifically alphalinolenic acid (ALA). It also contains omega 6 and omega 9 fatty acids. Omega fatty acids are the essential for our health, so the omega- $3 \mathrm{~s}$ and $6 \mathrm{~s}$ must be obtained through our diet or by supplementation. It is the best resources for additional human omega-3 polyunsaturated fatty acids (PUFAs). ALA (omega-3 fatty acid), is found in some other plant oils sources such as flaxseed (linseed), with lower amounts in walnut, canola, soy and animal sources like fish oil (cold water fish as salmon, cod and mackerel). 
Graph 1 Gas Chromatography report of perilla frutescens seeds oil

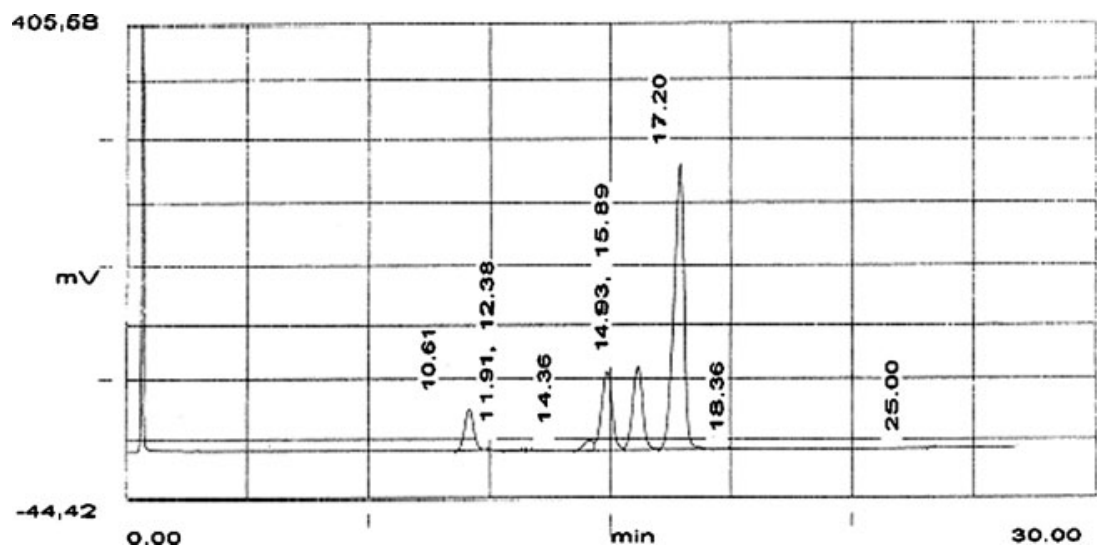

Perilla oil suppresses the production of chemical mediator in the allergy and inflammatory responses. These essential fatty acids have been associated with benefits in a wide range of inflammatory conditions, heart diseases, colitis/Crohn's disease, asthma, allergies, antimicrobial, anticancer etc. Perilla is also used for nausea, sunstroke, to induce sweating and as an antispasmodic. In vivo metabolism of polyunsaturated omega-3 fatty acids, it mainly exists in the form of DHA (docosahexaenoic acid) and EPA (eicosapentaenoic acid). These two specific omega-3 fatty acids metabolites are inserted in cell membranes throughout the body, where cellular machinery converts them into substances which prevent abnormal clotting, reduce inflammation, and relax blood vessels and improved ventilatory parameters (Lewis 2008; Talbott and Hughes 2006; Calder 2004; James et al. 2000; Chin et al. 1992; Mattson and Grundy 1985).

Other dietary sources of omega-3 fatty acids

\section{Botanical sources}

Flax seeds produce linseed oil, which has a very high omega -3 fatty acid content Six times richer than most fish oils in $n-3$, flax (or linseed) (Linum usitatissimum) and its oil are perhaps the most widely available botanical source of $n-3$. Flaxseed oil consists of approximately $55 \%$ ALA (alpha-linolenic acid). Flax, like chia, contains approximately three times as much $n-3$ as $n-6.15 \mathrm{~g}$ of flaxseed oil provides $8 \mathrm{~g}$ of ALA, which is converted in the body to EPA and then DHA at efficiency of $5-10 \%$ and $2-5 \%$, respectively (Azcona et al. 2008; Lewis 2008; Albert et al. 2002; Schacky and Dyerberg 2001; James et al. 2000; Mattson and Grundy 1985) (Tables 1 and 2).

\section{Animal sources}

Fish The most widely available source of EPA and DHA is cold water oily fish such as salmon, herring, mackerel, anchovies and sardines. Oils from these fish have a profile of around seven times as much omega-3 as omega-6. Other oily fish such as tuna also contain omega -3 in somewhat lesser amounts. Consumers of oily fish should be aware of the potential presence of heavy metals and fat-soluble pollutants like PCBs and dioxin which may accumulate up the food chain. Although fish is a dietary source of $n-3$ fatty acids, fish do not synthesize them; they obtain them from the algae or plankton in their diet (James et al. 2000; Renaud 2002; Chin et al. 1992).

Eggs Eggs produced by chickens fed a diet of greens and insects produce higher levels of $n-3$ fatty acids (mostly ALA) than chickens fed corn or soybeans. In
Table 1 Omega-3 content as the percentage of ALA in the seed oil (From Wikipedia, the free encyclopedia report)

\begin{tabular}{lllc}
\hline Common name & Alternative name & Linnaean name & \% of omega-3 \\
\hline Chia & chia sage & Salvia hispanica & 64 \\
Kiwifruit & Chinese gooseberry & Actinidia chinensis & 62 \\
Perilla & shiso & Perilla frutescens & 58 \\
Flax & linseed & Linum usitatissimum & 55 \\
Lingon berry & Cowberry & Vaccinium vitisidaea & 49 \\
Camelina & Gold-of-pleasure & Camelina sativa & 36 \\
Purslane & Portulaca & Portulaca oleracea & 35 \\
Black Raspberry & & Rubus occidentalis & 33 \\
\hline
\end{tabular}


Table 2 Omega -3 content as the percentage of ALA in the whole food (Wikipedia, the free encyclopedia)

\begin{tabular}{llc}
\hline Common name & Linnaean name & \% Omega-3 \\
\hline Flaxseed & Linum usitatissimum & 18.1 \\
Butternuts & Juglans cinerea & 8.7 \\
Hempseed & Cannabis sativa & 8.7 \\
Walnuts & Juglans regia & 6.3 \\
Pecan nuts & Carya illinoinensis & 0.6 \\
Hazel nuts & Corylus avellana & 0.1 \\
\hline
\end{tabular}

Some vegetables, too, contain a noteworthy amount of $n-3$, including strawberries and broccoli

addition to feeding chickens insects and greens, fish oils may be added to their diet to increase the amount of fatty acid concentrations in eggs. The addition of flax and canola seeds to the diet of chickens, both good sources of alpha-linolenic acid, increases the omega-3 content of the eggs (Azcona et al. 2008; Trebunová et al. 2007).

Meat The $n-6$ to $n-3$ ratio of grass-fed beef is about 2:1, making it a more useful source of $n-3$ than grain-fed beef, which usually has a ratio of $4: 1$. In most countries, commercially available lamb is typically grass-fed, and thus higher in $n^{-3}$ than other grain-fed or grain-finished meat sources. The omega- 3 content of chicken meat may be enhanced by increasing the animals' dietary intake of grains that are high in $n-3$, such as flax, chia, and canola (Azcona et al. 2008; Trebunová et al. 2007).

Seal oil Seal oil is a source of EPA, DPH, and DPA. According to Health Canada, it helps to support the development of the brain, eyes and nerves in children up to 12 years of age (Azcona et al. 2008; Trebunová et al. 2007).

\section{Other sources}

Milk and cheese from grass-fed cows may also be good sources of $n-3$. The microalgae Crypthecodinium cohnii and Schizochytrium are rich sources of DHA (22:6n-3) and can be produced commercially in bioreactors. This is the only source of DHA acceptable to vegans. Oil from brown algae (kelp) is a source of EPA. Walnuts are one of few nuts that contain appreciable $n-3$ fat, with approximately a 1:4 ratio of $n-3$ to $n-6$. Acai palm fruit also contains $n-3$ fatty acids. Omega- 3 is also found in soft gels in pharmacies and nowadays it is also found in combination with omega-6, omega-9 and shark liver oil(Azcona et al. 2008; Trebunová et al. 2007).

Chemistry of fatty acids

Perilla is an alternative source of fatty acids that contains both saturated and unsaturated (monosaturated and polyunsaturated) fatty acids. Fatty acids having more than one double bonds are termed as PUFAs. It contains saturated fatty acids mainly palmitic acid $5-7 \%$, stearic acid $1-3 \%$, monosaturated oleic acid $12-22 \%$, and poly saturated fatty acids linoleic acid 13-20\%, $\gamma$-linolenic acid $0-1 \%$, $\alpha$-linolenic acid 52-64\%, icosanoic acid $0-1 \%$. Increase in the number of double bonds progressively decreases the melting point. Unsaturated fatty acids are lower melting point than saturated fatty acids. Plant triglycerides have a large portion of unsaturated fatty acids such as oleic, lenoleic and linolenic acids. Animal triglycerides have high proportion of saturated fatty acids such as palmitic and stearic acids (Tables 3 and 4).

The carbon chain of saturated fatty acids posses zigzag configuration with the bond between carbon-carbon being $109^{\circ}$. The stearic acid $(18 \mathrm{C})$ depicted as fallows (Fig. 1).

Introduction of double bonds in oleic acid between carbon- 9 and carbon 10, causes bend in the molecule (Fig. 2).

Introduction of two double bonds (e.g. Linoleic acid) causes further bending of the hydrocarbon chain.

Geometrical isomerism occurs in fatty acids whose hydrocarbon has double bonds. Most unsaturated fatty acids occur in the relatively less stable isomeric form rather than more stable trans form (Fig. 3, Tables 3 and 4).

Nomenclature of fatty acids

The systemic nomenclature of fatty acids is derived from the name of its parent hydrocarbon by replacing its final e by

Table 3 Major saturated and saturated fatty acids

\begin{tabular}{llll}
\hline Saturated fatty acids & Molecular formula & Unsaturated fatty acids & Molecular formula \\
\hline Palmitic acid & $\mathrm{CH}_{3}\left(\mathrm{CH}_{2}\right)_{14} \mathrm{COOH}$ & Oleic acid & $\mathrm{CH}_{3}\left(\mathrm{CH}_{2}\right)_{7} \mathrm{CH}=\mathrm{CH}\left(\mathrm{CH}_{2}\right)_{7} \mathrm{COOH}$ \\
Stearic acid & $\mathrm{CH}_{3}\left(\mathrm{CH}_{2}\right)_{16} \mathrm{COOH}$ & Linoleic acid & $\mathrm{CH}_{3}\left(\mathrm{CH}_{2}\right)_{4} \mathrm{CH}=\mathrm{CHCH}_{2} \mathrm{CH}=\mathrm{CH}-\left(\mathrm{CH}_{2}\right)_{7} \mathrm{COOH}$ \\
Arachidic acid & $\mathrm{CH}_{3}\left(\mathrm{CH}_{2}\right)_{18} \mathrm{COOH}$ & Linolenic acid & $\mathrm{CH}_{3} \mathrm{CH}_{2} \mathrm{CH}=\mathrm{CHCH}_{2} \mathrm{CH}=\mathrm{CHCH} \mathrm{CH}_{2} \mathrm{CH}(\mathrm{CH})_{7} \mathrm{COOH}$ \\
& & Arachidonic acid & $\mathrm{CH}_{3}\left(\mathrm{CH}_{2}\right)_{3}\left(\mathrm{CH}_{2} \mathrm{CH}=\mathrm{CH}\right)_{4}(\mathrm{CH})_{3} \mathrm{COOH}$ \\
\hline
\end{tabular}


Table 4 Gas chromatography report of different components present in perilla oil

\begin{tabular}{lllc}
\hline Serial no. & Component & No. of double bonds & Retention time \\
\hline 1 & Palmitic acid & Saturated (0) & 10.61 \\
2 & Stearic acid & Saturated (0) & 14.36 \\
3 & Oleic acid & Monounsaturated (1) & 14.93 \\
4 & Linoleic acid & Polyunsaturated (2) & 15.89 \\
5 & Linolenic acid & Polyunsaturated (3) & 17.20 \\
\hline
\end{tabular}

oleic acid. Thus the names of saturated fatty acids end with the suffix anoic acid and those of unsaturated fatty acids with the suffix enoic acid. The numbering of carbon atoms in fatty acids is started at the carboxyl terminus and end methyl carbon is known as omega carbon atom (Figs. 4 and 5).

Various conventions are adopted for indicating the position of the double bonds. The most widely used are involve the use of the symbol $\Delta$ fallowed by superscript number. For example $\Delta^{9}$ means that there is a double bound between carbon 9 and carbon 10. Alternatively the position of the double bond is indicated by the numerals as in case simple alkenes. Lastly note that total number of carbon atoms and number of position(s) of double bond(s) is again indicated by convention. Examples, the symbol 18;0 denote a C18 fatty acid with no double bonds, the symbol 18: 1; 9 denote a C 18 fatty acid with a double bond between carbon 9 and carbon 10 and the symbol 18: 2; 9,12 denote a C 18 fatty acid with two double bonds between $\mathrm{C} 9$ and $\mathrm{C} 10$ and between $\mathrm{C} 12$ and 13 (Renaud 2002; Mattson and Grundy 1985) (Table 5).

\section{Definition of omega fatty acids}

The names "omega 3" or "omega 6" or "omega 9" fatty acids refer to where a double bond occurs in the fatty acid molecule. The terms "omega" or "n minus" refer to the position of the double bond of the fatty acid closest to the methyl end of the molecule. Thus, oleic acid, which has its double bond 9 carbons from the methyl end, is considered an omega-9 (or an $n-9$ ) fatty acid. Similarly, linoleic acid, common in vegetable oils, is an omega-6 (n-6) fatty acid because its second double bond is 6 carbons from the methyl end of the molecule (i.e., between carbons 12 and 13 from the carboxyl end). Omega 3 and omega 6 fatty acids are "essential fatty acids", meaning that these fatty acids cannot synthesized by body itself. Instead, we must include them in our diet or through supplements to meet our body demands. Omega 9 fatty acids are "conditionally essential", which means that if we have the other fatty acids in our diet, then our body can manufacture omega 9 fatty acids. Otherwise, omega 9 fatty acids must be consumed or supplemented as well. DHA (docosahexaenoic acid) and EPA (eicosapentaenoic acid) are the two specific omega 3 fatty acids found in fish oil such as cold water fish as salmon, cod and mackerel (Green et al. 2007; Calder 2004; Renaud 2002; Chin et al. 1992; Ip et al. 1996; Mattson and Grundy 1985).

Importance of omega fatty acids

Among plant oils, the balance between omega-3, omega-6 and omega 9 fatty acids must dictate which oil is chosen. Oils which predominate in omega- 3 component would be most likely to promote health, only perilla and flax seed (vegetable) oil predominate in omega-3 fatty acid. Most would actually contribute to the imbalance of omega- 6 fatty acids because they contain more omega- 6 than omega-3. Any amount of omega-9 is beneficial, but in balancing these fatty acids, the omega-3 component is the most important.

The most common omega 6 fatty acid is linoleic acid. When omega 6 fatty acids are consumed in the diet, they are inserted in the cell membranes, where the same cellular machinery converts them into substances which promote abnormal clotting and increase inflammation. While Omega-3 fatty acids are beneficial to improved cardiovascular health, and certain types of cancers, as well as enhancing the immune system (Lewis 2008; Reisman et al. 2006; Talbott and Hughes 2006; Calder 2004; Albert et al. 2002; James et al. 2000; Longvah et al. 2000; Lee et al. 1994; Narisawa et al. 1994; Thompson et al. 1997; Chin et al. 1992; Kromann and Green 1980).

Balance of omega-3 \& 6 is key for normal immune function

Many health issues depend on a proper balance of omega 3 and omega 6 fatty acids. While omega 6 fatty acids are necessary for normal immune function and clotting, too much omega 6 fatty acid may promote abnormal clotting and an overactive immune system. It is believed that our ancestors evolved on a diet where these two omega fatty acids were approximately equal. However, modern diets usually have up to 20 times more omega 6 fatty acids than omega 3 fatty acids. Many of the chronic degenerative



Fig. 1 Zigzag configuration of unsaturated stearic acid (18 C) 


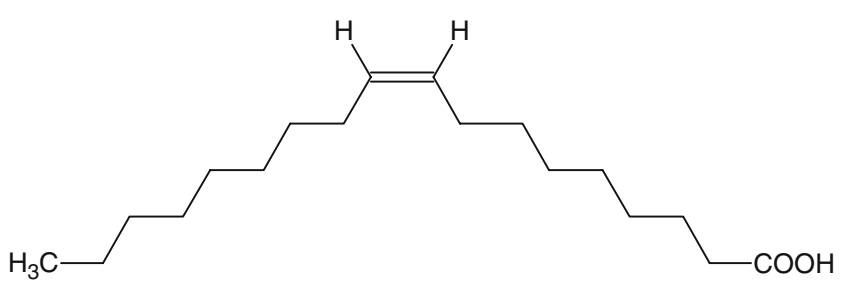

Fig. 2 Configuration of monosaturated oleic acid (C 18)

diseases we experience today are believed to have their origins in an imbalance of omega 3 and omega 6 fatty acids in our diet. This necessitates that $n-3$ and $n-6$ be consumed in a balanced proportion; healthy ratios of $n-6: n-3$ range from 1:1 to 4:1. (Renaud 2002; Mattson and Grundy 1985).

Cardio-vascular benefits

Heart disease is the one of the most common diseases nowadays due to current life style and eating habits. Certain population studies have shown that a diet high in omega-3 fatty acids, specifically EPA and DHA found in fish oil or metabolized product of ALA (perilla oil) can help to prevent heart disease. Omega-3 fatty acid (ALA), through the body's metabolic pathway, can be converted into EPA and DHA at a rate of roughly $7-10 \%$. The research proved that when using omega-3 rich perilla oil instead of soybean oil, the subjects increased omega-3 levels in their blood, which may lead to prevention of coronary heart disease and decrease blood clotting (Lewis 2008; Calder 2004; Schacky and Dyerberg 2001).

In different cells of the body, the cellular machinery makes different things. In platelets, the cell products in the blood which aid in clotting, omega 6 fatty acids are converted to thromboxane A2 (TXA2). This makes the platelets more likely to burst (degranulate), releasing their clotting substances and cell messengers. These cell messengers constrict blood vessels and tell other platelets to burstcausing a clotting cascade. On the other hand, when omega 3 fatty acids are used in the same machinery in platelets, thromboxane A3 (TXA3) is made, which is inactive. If you have been cut or injured, you want the bleeding to stop with the help of platelets. However, if you have not been cut or injured, clotting is abnormal and may block flow to areas which need it-causing a

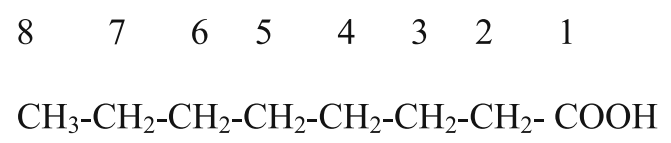

$\omega \quad \delta \quad \gamma \quad \beta \quad \alpha$

Fig. 3 Geometrical isomerism of unsaturated fatty acids

\section{$\begin{array}{llllllll}8 & 7 & 6 & 5 & 4 & 3 & 2 & 1\end{array}$ \\ $\mathrm{CH}_{3}-\mathrm{CH}_{2}-\mathrm{CH}_{2}-\mathrm{CH}_{2}-\mathrm{CH}_{2}-\mathrm{CH}_{2}-\mathrm{CH}_{2}-\mathrm{COOH}$}

$\omega \quad \delta \quad \gamma \quad \beta \quad \alpha$

Fig. 4 Numbering of carbon atoms in fatty acids

heart attack or stroke. In white blood cells (WBC's), the infection fighting cells of the body, omega 6 fatty acids make more inflammatory substances. These substances include leukotriene B4, (LTB4), which is a cell messenger responsible for inflammation throughout the body. It is a "call to arms" for other WBC's. LTB4 even tells certain WBC's to get into the wall of the blood vessel. LTB4 actually causes these WBC's to absorb oxidized LDL cholesterol (cholesterol plaque is formed). In contrast, when omega 3 fatty acids are used in the same cellular machinery, leukotriene B5 (LTB5) is made. LTB5 is anti-inflammatory. Health demands normal functioning of both systems (Bemelmans et al. 2002; de Lorgeril et al. 1999; Thompson et al. 1997; Lee et al. 1994).

Anti-inflammatory and rheumatoid arthritis benefits

Perilla oil is rich in the omega- 3 fatty acids, on metabolism gives eicosapentaenoic acid (EPA) and docosahexaenoic acid (DHA), which can displace arachidonic acid (AA) from

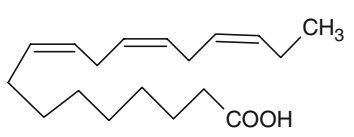

Alpha-linolenic acid [ALA]

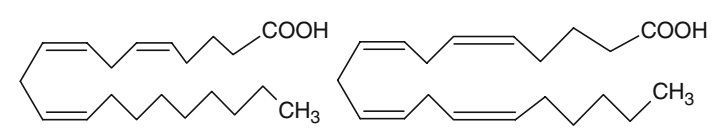

Eicosatrienoic Acid Arachidonic Acid [AA]

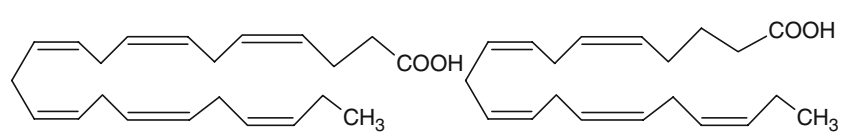

Docosahexaenoic acid [DHA] Eicosapentaenoic Acid [EPA]

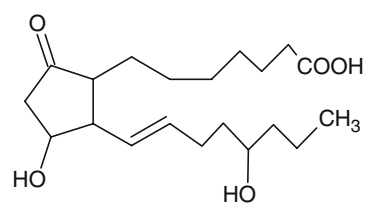

Prostaglandin [PGE]

Fig. 5 Structure of alfa linolenic acid (ALA) arachidonic acid (AA), Docosahexaenoic acid (DHA) prostaglandin (PGE) and eicosapentaenoic acid (EPA) 
Table 5 List of several different names for the most common $n-3$ fatty acids

\begin{tabular}{lll}
\hline Common name & Lipid name & Chemical name \\
\hline Alfa Linolenic Acid (ALA) & $18: 3(n-3)$ & all-cis-9,12,15-octadecatrienoic acid \\
Eicosapentaenoic acid (EPA) & $20: 5(n-3)$ & all-cis-5,8,11,14,17-eicosapentaenoic acid \\
Docosahexaenoic acid (DHA) & $22: 6(n-3)$ & all-cis-4,7,10,13,16,19-docosahexaenoic acid \\
\hline
\end{tabular}

cell membranes. These omega-3 fatty acids are also released with AA by phospholipases and act as substrate inhibitors of conversion of AA by cyclo-oxygenases (COX) and the terminal synthases to the pro-inflammatory oxygenated inflammatory mediators known as eicosanoids. EPA is structurally identical to AA with the exception of its additional $n-3$ double bond and can be converted to eicosanoids that resemble eicosanoids. In addition to these effects on inflammatory eicosanoid synthesis, perilla oils have been shown to reduce the production of the inflammatory cytokines IL-1 $\beta$ and TNF $\alpha$ by monocytes stimulated in vitro. These cytokines are important effector molecules in inflammatory responses and $\mathrm{TNF} \alpha$ blocking agents are now used widely to treat rheumatoid disease that has proven refractory to less expensive therapies. In vitro studies have also shown inhibition of release of the metalloproteinases that are implicated in the tissue damage that is the hallmark of rheumatoid arthritis and other inflammatory diseases (Osakabe et al. 2005, 2004; Banno et al. 2004; James et al. 2000).

It has been reported that conversion of ALA to EPA and further to DHA in humans is limited, but varies with individuals. Women have higher ALA conversion efficiency than men, probably due to the lower rate of utilization of dietary ALA for beta-oxidation.

Perilla oil reduces recourse to NSAIDs for analgesia in rheumatoid arthritis and thereby reduces risk for upper GI haemorrhage. Perilla oil contrasts with the highly selective COX-2 inhibitor rofecoxib, which has been associated with increased serious cardiovascular events, by reducing risk for these events. The result is fewer AA derived eicosanoids with production of homologous metabolites products such as PGE1 (one less double bond than AA derived PGE2). ALA rich oils appear to reduce symptoms in rheumatoid arthritis but available evidence is far less than that for perilla oil in rheumatoid arthritis (Osakabe et al. 2005; Banno et al. 2004; Calder 2004; James et al. 2000; Borchers et al. 1997).

\section{Cancer benefits}

Similarly, studies in animals have found that omega- 3 fatty acids suppress cancer formation, but at this time there is no direct evidence for protective effects in humans. A group of isomers of the essential fatty acid linoleic acid, "conjugated linoleic acid" (CLA), appear to have both anticarcinogenic and antiatherogenic properties and may affect body composition. CLA differs from linoleic acid by the position and geometric configuration of one of its double bonds (Chin et al. 1992). Animal studies have indicated that CLA reduces the incidence of tumors induced by carcinogens (Ip et al. 1996; Thompson et al. 1997). In addition to its anticarcinogenic properties, CLA appears to be antiatherogenic as well (Lee et al. 1994). Saturated fatty acids have not been found to have any specific effects on carcinogenesis. On a positive note, recent studies have shown that conjugated linoleic acid, appears to be unique among fatty acids because low levels in the diet produce significant cancer protection (Banno et al. 2004; Osakabe et al. 2004; Caughey et al. 1996; Narisawa et al. 1994; Chin et al. 1992).

\section{Antiallergic benefits}

Numerous studies have shown that perilla seed oil suppressed a wide range of allergic mediators in experimental animals. These findings raise the potential for perilla oil to be effective in reducing allergic hypersensitivity in humans. Allergic mediators were decreased in the perilla oil (ALA). The perilla oil group also showed benefits in terms of lung function, breathing parameters and may be useful for the treatment of asthma. Perilla oil for reduction in asthma symptoms due to the alpha-lineolic acid effects on leukotrienes as well as use of perilla extract for seasonal allergies (Gediminas et al. 2008; Reisman et al. 2006; Osakabe et al. 2005, 2004; Ragažinskienė et al. 2004; Borchers et al. 1997).

\section{Brain health benefits}

PUFAs ( $n-3$ and $n-6,3000 \mathrm{mg}$ a day), PUFAs plus multivitamins and minerals or placebo supplementation in children with learning and behavioral problems (Green et al. 2007). Omega-3 exerts neuroprotective action in Parkinson's disease, exhibit a protective effect (much like it did for Alzheimer's disease as well). The high doses of omega-3 given to the experimental group completely prevented the neurotoxin-induced decrease of dopamine that ordinarily occurs. Since Parkinson's is a disease caused by disruption of the dopamine system, this 
protective effect exhibited could show promise for future research in the prevention of Parkinson's disease (Talbott and Hughes 2006).

\section{Numerous applications}

Not only does perilla contain the omega-3 ALA, but perilla is also a rich source of phenolic compounds, flavanoids and anthocyanins known for their antioxidant properties. Rosemarinic acid, luteolin, chrysoeriol, quercetin, catcehin and apigenin are a few of the phenolic compounds found in perilla oil. These antioxidants may also be involved in allergy, antimicrobial, cardiovascular and cancer prevention along with the omega-3 fatty acids. Perilla seeds contain polyphenols luteolin showed the strong antimicrobial effect than other phenolic compounds (Gediminas et al. 2008; Yamamoto and Ogawa 2002).

Research has observes that populations consuming diets high in ALA, reduction in the death rate due to coronary heart disease. As perilla seed oil is one of the richest sources of ALA, the research is clear more perilla equals improved heart health. Other studies suggest that omega-3 fatty acids are useful in the treatment of cancer, inflammatory, colitis/Crohn's disease, arthritis and various skin conditions. Anti-inflammatory doses of perilla oil have been shown to reduce the hypertensive and nephrotoxic effects of cyclosporin. It prevent the formation of LTB4 have been used in treating asthma, rheumatoid arthritis, colitis, lupus, multiple sclerosis, and psoriasis (Osakabe et al. 2005; Banno et al. 2004; Calder 2004; Albert et al. 2002; Bemelmans et al. 2002; de Lorgeril et al. 1999; Borchers et al. 1997; Caughey et al. 1996).

Perilla oil can be used as a tasty addition to salad dressings, dips, yogurt, hot cereal, blender drinks and other foods. Many individuals enjoy the flavor of perilla oil taken by the teaspoonful. Perilla oil is also available in an easy to swallow, soft gel form. The nourishment supplied by $2.1 \mathrm{~g} \alpha$-linolenic acid equal that by $30 \mathrm{~g}$ fish oil. To receive the numerous health benefits that perilla oil has to offer, a suggested dosage is $3 \mathrm{~g}$ per day (Green et al. 2007; Reisman et al. 2006; Talbott and Hughes 2006; Yamamoto and Ogawa 2002; Longvah et al. 2000; Schacky and Dyerberg 2001; Lee et al. 1994).

Health risks

Known or suspected risks of EPA and DHA omega -3 fatty acids may include the possibility of: Increased bleeding if overused (normally over $3 \mathrm{~g}$ per day) by a patient who is also taking aspirin or warfarin. However, this is disputed. Hemorrhagic stroke (only in case of very large doses). Reduced glycemic control among diabetics (Lewis 2008; Kromann and Green 1980).
Cardiac risk

Persons with congestive heart failure, chronic recurrent angina pectoris or evidence that their heart is receiving insufficient blood flow are advised to talk to their doctor before taking $n-3$ fatty acids. There have been concerns if such persons take $n-3$ fatty acids or eating foods that contain them in substantial amounts. In a recent large study, $n-3$ fatty acids on top of standard heart failure therapy produced a small but statistically significant benefit in terms of mortality and hospitalization (Ornish 2006).

\section{Daily values}

According to food and nutrition Board (2005) acceptable intake (AI) for $n-3$ is $1.6 \mathrm{~g} /$ day for men and $1.1 \mathrm{~g} /$ day for women while the acceptable macronutrient distribution range AMDR is $0.6 \%$ to $1.2 \%$ of total energy. "A growing body of literature suggests that higher intakes of $\alpha$-linolenic acid (ALA), eicosapentaenoic acid (EPA), and docosahexaenoic acid (DHA) may afford some degree of protection against coronary heart disease. Because the physiological potency of EPA and DHA is much greater than that for $\alpha$-linolenic acid, it is not possible to estimate one AMDR for all $n-3$ fatty acids. Approximately $10 \%$ of the AMDR can be consumed as EPA and/or DHA." The FDA recommends that total dietary intake of $n-3$ fatty acids from fish not exceed $3 \mathrm{~g}$ per day, of which no more than $2 \mathrm{~g}$ per day are from nutritional supplements (Lewis 2008; Talbott and Hughes 2006; Bemelmans et al. 2002; Schacky and Dyerberg 2001; Chin et al. 1992; Mattson and Grundy 1985).

\section{Discussion}

Research showed that the low incidence of heart attacks even their diet was so high in fat. When the fats in the diet were studied, it appeared that omega 3 fatty acids, found in high levels in their food, provided protection from heart attacks. Perilla oil to be potentially benefits include protecting the heart (reduced blood clotting), reducing allergic symptoms and asthma, acting as a pain reliever and anti-inflammatory, stimulating immune function, intestinal dryness and constipation. Perilla oil to be potentially benefits to prevent and cure different ailments but little health risk was also reported.

Perilla is used as culinary and medicinal herb in many part of Asia. Perilla seed supplies the polyphenolic ingredients luteolin and rosmarinic acid- two substances that have a powerful impact on decreasing allergy symptoms. ALA in vivo decreases the blood pressure and reduced the cholesterol and glyceride contents in the blood. It controls hematoblastic aggregation and thrombi reduc- 
tion. ALA has definite inhibitory action on the growth and metabolism of breast and colon cancer.

\section{Conclusion}

Yet if we increase our intake of omega-3 fatty acids, we may prevent the formation of these inflammatory substances and actually increase the production of anti-inflammatory substances. Most important to people concerned about cardiovascular disease, omega 3 fatty acids have been shown to improve blood pressure and vascular resistance, lower triglycerides, prevent abnormal heart rhythm and sudden cardiac death, and may reduce the risk of heart attack. The high content of omega-3 fatty acids, the absence of mercury risk from fish sources, and the beneficial effects of perilla oil is the best supplement to achieve balance in these valuable fatty acids. Although perilla has been around for centuries, is has recently as a viable vegetarian omega-3 alternative for nutrition and health. Linolenic acid is also a precursor of a special group of prostaglandins.

Person, those who are vegetarian or dislike the taste of fish, have a fish allergy or are just unable to tolerate fish oil due to reflux, vegetarian sources (Perilla oil) of omega-3 fatty acids are preferable. Plant based omega-3s including perilla, flax, purslane, and walnut will provide essential fats for necessity of our body. Now that the low fat diet craze is over, healthy fats from sources such as perilla oil, fish oils, flaxseed oil, hemp oil, and evening primrose oil are making their way back into the diets. More clinical evidence is required before recommendations can be made for any clinical usage of perilla.

Open Access This article is distributed under the terms of the Creative Commons Attribution Noncommercial License which permits any noncommercial use, distribution, and reproduction in any medium, provided the original author(s) and source are credited.

\section{References}

Albert CM, Campos H, Stampfer M et al (2002) Blood levels of longchain $\mathrm{n}-3$ fatty acids and the risk of sudden death. N Engl J Med 346(15):1113-1118

Azcona JO, Schang MJ, Garcia PT, Gallinger C, Ayerza R, Coates W (2008) Omega-3 enriched broiler meat: the influence of dietary alpha-linolenic omega-3 fatty acid sources on growth, performance and meat fatty acid composition. Can J Anim Sci Ott Ont Can 88:257-269

Banno N, Akihisa T, Tokuda $\mathrm{H}$ et al (2004) Triterpene acids from the leaves of Perilla frutescens and their anti-inflammatory and antitumor-promoting effects. Biosci Biotechnol Biochem 68 (1):85-90
Bemelmans W, Broer J, Feskens E, Smit A, Muskiet F, Lefrandt J, Bom V, May J, Meybook-de Jong B (2002) Effect of an increased intake of a-linolenic acid and group nutritional education on cardiovascular risk factors: the Mediterranean Alpha-linolenic Enriched Groningen Dietary Intervention (MARGARIN) study. Am J Clin Nutr 75(2):221-227

Borchers AT, Hackman RM, Keen CL (1997) Complementary medicine: a review of immunomodulatory effects of Chinese herbal medicines. Am J Clin Nutr 66(6):1303-1312

Calder PC (2004) n-3 Fatty acids and cardiovascular disease: evidence explained and mechanisms explored. Clin Sci (Lond) 107:1-11

Caughey GE, Mantzioris E, Gibson RA, Cleland LG, James MJ (1996) The effect on human tumor necrosis factor and interleukin- $1 \mathrm{~b}$ production of diets enriched in n-3 fatty acids from vegetable oil or fish oil. Am J Clin Nutr 63:116-122

Chin SF, Liu W, Storkson JM, Ha YL, Pariza MW (1992) Dietary sources of conjugated dienoic isomers of linoleic acid, a newly recognized class of anticarcinogens. J Food Compos Anal 5:185-197

de Lorgeril M, Salen P, Martin JL, Monjaud I, Delaye J, Mamelle N (1999) Mediterranean diet, traditional risk factors, and the rate of cardiovascular complications after myocardial infarction. Circulation 99:779-785

Gediminas Z, Jonas Z, Renata S, Genovaitè S, Egidijus K (2008) Effect of Perilla frutescens aqueous extract on free radical production by human neutrophil leukocytes. Medicina (Kaunas) 44(9):699-705

Green KN, Martinez-Coria H, Khashwji H, Hall EB, Yurko-Mauro KA, Ellis L, LaFerla FM (2007) Dietary docosahexaenoic acid and docosapentaenoic acid ameliorate amyloid- $\beta$ and tau pathology via a mechanism involving presenilin 1 levels. J Neurosci 27 (16):4385-4395

Ip C, Briggs SP, Haegele AD, Thompson HJ, Storkson J, Scimeca JA (1996) The efficacy of conjugated linoleic acid in mammary cancer prevention is independent of the level or type of fat in the diet. Carcinogenesis 17:1045-1050

James MJ, Gibson RA, Cleland LG (2000) Dietary polyunsaturated fatty acids and inflammatory mediator production. Am J Clin Nutr 71:343S-348S

Kromann N, Green A (1980) Epidemiological studies in the Upernavik district, Greenland. Incidence of some chronic diseases 1950-1974. Acta Med Scand 208(5):401-406

Lee KN, Kritchevsky D, Pariza MW (1994) Conjugated linoleic acid and atherosclerosis in rabbits. Atherosclerosis 108:19-25

Lewis CJ (2008) Letter regarding dietary supplement health claim for omega-3 fatty acids and coronary heart disease, http://www. cfsan.fda.gov/ dms/ds-ltr11.htmland "Letter Regarding Dietary Supplement Health Claim for Omega-3 Fatty Acids and Coronary Heart Disease". U. S. Food and Drug Administration via Internet Archive

Longvah T, Deosthale YG, Kumar PU (2000) Nutritional and short term toxicological evaluation of Perilla seed oil. Food Chem $70: 13-16$

Mattson FH, Grundy SM (1985) Comparison of effects of dietary saturated, monounsaturated, and polyunsaturated fatty acids on plasma lipids and lipoproteins in man. J Lipid Res 26:194-202

Narisawa T, Fukaura Y, Yazawa K, Ishikawa C et al (1994) Colon cancer prevention with a small amount of dietary perilla oil high in alpha linolenic acid in an animal model. Cancer 73(8):2069-2075

Ornish D (2006) "The Dark Side of Good Fats". Newsweek: p 2. Food and Nutrition Board, Institute of Medicine of the National Academies (2005), 423, 770

Osakabe N, Yasuda A, Natsume M, Yoshikawa T (2004) Rosmarinic acid inhibits epidermal inflammatory responses: anticarcinogenic 
effect of Perilla frutescens extract in the murine two-stage skin model. Carcinogenesis 25(4):549-557

Osakabe N, Takano H, Sanbongi C et al (2005) Anti-inflammatory and anti-allergic effect of rosmarinic acid (RA); inhibition of seasonal allergic rhinoconjunctivitis (SAR) and its mechanism. Biofactors 21:127-131

Ragažinskienė O, Gailys V, Jankauskienė K, Šimonienė G, Jurkštienė V (2004) Krūminè perilè. (Perilla frutescens (L.) Britton)-perspektyvus imunomoduliatorius. Common perilla (Perillafrutescens (L.) Britton.) as a perspective immunomodulator. Medicina (Kaunas) 40(3): $220-224$

Reisman J, Schachter HM, Dales RE, Tran K, Kourad K, Barnes D, Sampson M, Morrison A, Gaboury I, Blackman J (2006) Treating asthma with omega-3 fatty acids: where is the evidence? A systematic review. Med BMC Complement Altern Med 6(26):1-8

Renaud SC (2002) The importance of the ratio of omega-6/omega-3 essential fatty acids. Biomed Pharmacother 56(8):365-379
Schacky C, Dyerberg J (2001) Omega-3 fatty acids. From Eskimo to clinical cardiology — what took us so long? World Rev Nutr Diet 88:90-99

Talbott SM, Hughes K (2006) Perilla seed oil (Perilla frutescens). In: The health professional's guide to dietary supplements. Lippincott Williams \& Wilkins, Baltimore, MD, pp 258-260

Thompson H, Zhu Z, Banni S, Darcy K, Loftus T, Ip C (1997) Morphological and biochemical status of the mammary gland is influenced by conjugated linoleic acid: implication for a reduction in mammary cancer risk. Cancer Res 57:5067-5072

Trebunová A, Vasko L, Svedová M, Kastel’ R, Tucková M, Mach $\mathrm{P}$ (2007) The influence of omega-3 polyunsaturated fatty acids feeding on composition of fatty acids in fatty tissues and eggs of laying hens. Dtsch Tierärztl Wochenschr 114(7):275-279

Yamamoto H, Ogawa T (2002) Antimicrobial activity of perilla seed polyphenols against oral pathogenic bacteria. Biosci Biotechnol Biochem 66:921-924 